\title{
Prediction of Patients with Ovarian Hyperstimulation Syndrome in Gnrh-A Prolonged Protocol : A Retrospective Study
}

Yang Zexing ( $\sim$ yangzexingkm@163.com )

Kunming Medical University First Affilliated Hospital https://orcid.org/0000-0002-0311-798X

\section{Wen Ya}

Kunming Medical University First Affilliated Hospital

Limei He

Kunming Medical College: Kunming Medical University

Yuhang Zheng

Centers for Disease Control

Meng Rao

Kunming Medical University First Affilliated Hospital

Huawei Wang

Kunming Medical University Affiliated Stomatology Hospital

Research

Keywords: ovarian hyperstimulation syndrome, ART, GnRH-a Prolonged Protocol

Posted Date: November 1st, 2021

DOI: https://doi.org/10.21203/rs.3.rs-1006958/v1

License: (9) (i) This work is licensed under a Creative Commons Attribution 4.0 International License. Read Full License 


\section{Abstract}

Background: OHSS (ovarian hyperstimulation syndrome) is a life-threatening complication and most common adverse effect of fertility treatment

Objectives: To investigate associated risk factors of ovarian hyperstimulation syndrome (OHSS) in stimulated ovarian cycles with assisted reproductive technology, may provide guidance for clinicians on how to prevent OHSS.

Methods: A Logistic regression analysis was conducted in 336 patients who had completed IVF-ICSI/ET cycles between April 2019 and April 2020 in the first affiliated hospital of kunming medical university. Receiver-operating characteristic (ROC) curves for OHSS were calculated for each predictor using cut-off values. Area under the curve (AUC) analysis and logistic regression models were performed to compare the performance of laboratory biomarkers.

Results: According to clinical diagnosis, 61 cycles developed OHSS of 336 cycles, with an incidence of $18.15 \%$. Patients were graded according to their clinical symptoms and severity, including 27 cycles of moderate OHSS (8.04\%) and 8 cycles of severe OHSS (2.38\%). The cut-off value of AMH, E2 on HCG day, egg retrieved to predict moderate and severe OHSS were $7.495 \mathrm{ng} / \mathrm{ml}, 4828 \mathrm{pg} / \mathrm{ml}$ and $19.5 \mathrm{in} \mathrm{GnRH}-\mathrm{a}$ Prolonged Protocol, with sensitivities of $77.0 \%, 67.2 \%, 80.3 \%$, and specificity of $68.0 \%, 70.9 \%, 81.5 \%$ respectively. The area under the curve (AUC) values of AMH, E2 on HCG day, Number of oocytes retrieved to predict moderate and severe OHSS were $0.754,0.738,0.876$, respectively. And the AUC value of combined index to predict moderate and severe OHSS was 0.898 , achieved the highest AUC with $85.2 \%$ sensitivity and $83.6 \%$ specificity.

Conclusions:AMH, E2 day of HCG and number of eggs obtained have a good predictive effect on the occurrence of OHSS, and the predictive ability is further improved after the combination of the three indicators.

\section{Introduction}

ovarian hyperstimulation syndrome (OHSS) is a serious life-threatening iatrogenic complication, it may occur spontaneously during pregnancy. The main pathophysiological mechanism is the increase of capillary permeability[1]. HCG is considered to be the main triggering factor of the syndrome[2]. Increased vascular permeability leads to fluid transfer from intravascular space to extracellular space. Women with high estradiol level, large number of follicles or oocytes retrieved, and polycystic ovary syndrome (PCOS) patients seem to have a particularly high risk of developing OHSS[3]. However, early identification of potential risk factors of OHSS and clinical intervention can significantly reduce the incidence of OHSS. Therefore, early prediction of OHSS occurrence and timely intervention measures are important links to improve the safety of assisted reproductive technique (ART), With the increasing use of ART, fertility physicians need to be equipped with the knowledge to control the occurrence of OHSS. But the indicators that predicted the occurrence of OHSS were different, some studies suggest that AFC and basal E2 are 
strong predictors of OHSS[4], other risk factors of OHSS include age, low body mass index (BMI), polycystic ovary syndrome (PCOS), and previous OHSS history, serum anti-Müllerian hormone (AMH), antral follicle count (AFC), estradiol $\left(E_{2}\right)$ on the day of human chorionic gonadotropin (hCG) injection and the number of oocytes retrieved were also predictors of OHSS[5]. A single indicator has limitations in the sensitivity and specificity of OHSS prediction, but the combination of multiple indicators may improve the prediction of disease[6, 7], however, there is no study on the prediction of OHSS with multiple indicators.

GnRH-a Prolonged Protocol was considered have higher cumulative live birth rate in POSEIDON group 4 patients with higher anti-Müllerian hormone levels (anti-Müllerian hormone $\geq 0.785 \mathrm{ng} / \mathrm{mL}$ ) than gonadotropin-releasing hormone antagonist protocol[8], and has higher CLBR, normal fertilization rate and number of available embryo than GnRH-ant group in a RCT research including 18853 women from China[9],but didn't increase the risk of OHSS compared to GnRH antagonist protocol[10]. However, at present, there is no study on the risk factors of OHSS in this regimen.

The aim of this study was to identify risk factors that can predict OHSS in patients with GnRH-a Prolonged Protocol, better avoid OHSS occurrence in this regimen.

\section{Materials And Methods}

\section{Study Design and Participants}

This is a retrospective study including 336 cases received IVF/ ICSI treatment using GnRH-a Prolonged Protocol from April 2019 to April 2020 in the First Affiliated Hospital of Kunming Medical University.

\section{Inclusion criteria}

1. The eligible women received their cycles of IVF or ICSI because of tubal factors, male factors, or a combination of both.

2. IVF/ICSI-fresh embryo transfer (ET) and an age $\leq 40$ years.

3. The first cycle of the same patient.

\section{Exclusion criteria}

a. Patients with a diagnosis of recurrent spontaneous abortion (RSA)

b. Couples (either the male or female) with chromosomal abnormalities and a history of cancer were excluded.

c. Preimplantation genetic testing (PGT)

d. Missing clinical data

e. Female age $>40$ years

f. Sperm donor cycle

\section{Diagnosis of PCOS}


Polycystic ovary syndrome diagnosed by modified Rotterdam criteria (Rotterdam ESHRE/ASRMSponsored PCOS Consensus Workshop Group 2004,)[11]

\section{Classification of OHSS symptoms}

Ttraditionally classified into mild, moderate, severe and critical, based on OHSS prevention and treatment guideline of American Society for Reproductive Medicine[12].

Mild-OHSS: abdominal distension/discomfort, mild nausea/vomiting, mild dyspnea, diarrhea, enlarged ovaries, and no important alterations [12]:

Moderate-OHSS: mild features, ultrasonographic evidence of ascites, hemoconcentration (Hct $>41 \%)$, and elevated WBC (>15,000 mL)[12],

Severe-OHSS: mild and moderate features, clinical evidence of ascites, hydrothorax, severe dyspnea, oliguria/anuria, intractable nausea/vomiting, severe hemoconcentration (Hct $>55 \%), W B C>25,000 \mathrm{~mL}$, $\mathrm{CrCl}<50 \mathrm{~mL} / \mathrm{min}, \mathrm{Cr}>1.6 \mathrm{mg} / \mathrm{dL}, \mathrm{Na}+<135 \mathrm{mEq} / \mathrm{L}, \mathrm{K}+>5 \mathrm{mEq} / \mathrm{L}$, and elevated liver enzymes, critical: low blood/central venous pressure, pleural effusion, rapid weight gain ( $>1 \mathrm{~kg}$ in $24 \mathrm{~h}$ ), syncope, severe abdominal pain, venous thrombosis, anuria/acute renal failure, arrhythmia, thromboembolism, pericardial effusion, massive hydrothorax, arterial thrombosis, adult respiratory distress syndrome, sepsis, and worsening of findings[12].

\section{Measurements and laboratory analyses}

Gonadal hormone concentrations and AMH measurements were performed on the serum of each subjects. Level of AMH will be examined using a Gen 2 ELISA kit (Beckman Coulter, Inc. Brea, CA, USA). Serum was stored at $-70^{\circ} \mathrm{C}$. A combined gas chromatographic negative ionization tandem mass spectrometry and liquid chromatographic electrospray tandem mass spectrometry bioanalytical method was used to measure Gonadal hormone (Taylor Technology, Princeton NJ).

\section{Early-Follicular Phase Gonadotropin-Releasing Hormone Agonist (GnRH-a) Prolonged Protocol}

One depot of $3.75 \mathrm{mg} \mathrm{GnRH}$-a was injected on day 2 of the menstrual cycle, 35 days later, pituitary downregulation was confrmed (no follicular diameter $>8 \mathrm{~mm}, \mathrm{E} 2<50 \mathrm{pg} / \mathrm{ml}$ ), Gonadotropin ( $\mathrm{FFH}-\mathrm{G}$-Gonal-f Merck Serono, Germany) was used at an initiative dose ranging from 150-225 U. The dose of Gn was adjusted according to the size, counts of follicles and hormone levels. When 2 dominant follicles reached $18 \mathrm{~mm}$ in diameter, or three follicles $=>17 \mathrm{~mm}$, human chorionic gonadotrophin (HCG, 5000-10,000 IU, Lizhu Pharmaceutical Trading Co., German) was administered for trigger ovulation[13].

If number of oocytes retrieved $\geq 15$, the serum E2 $\geq 5000 \mathrm{pg} / \mathrm{ml}$ on hCG trigger day or the serum E2 $\geq 1500 \mathrm{pg} / \mathrm{ml}$ on the second day after oocyte retrieval, or OHSS manifestations such as nausea, vomiting, abdominal pain and distension appeared, patients would receive a "freeze-all" approach instead of fresh embryo transfer to prevent late OHSS[12]. 
There were 61 cases of OHSS and 275 cases of non-OHSS. The database was collected after the approval of the medical ethics committee. All data were collected from the electronic medical record system and adjusted by at least two authors.

All of the included patients read and signed the informed consent form. This retrospective study was approved by the Ethics Committee of the First Affiliated Hospital of Kunming Medical University. All the treatments in the present study were performed strictly in accordance with the Declaration of Helsinki for Medical Research.

\section{Statistical analysis}

All analyses were performed using SPSS 22.0 statistical software package (SPSS Inc, Chicago, IL). According to the distribution of data types to choose the appropriate statistical methods to analyze the differences between groups, two independent groups were assessed using Student's t-test or the Wilcoxon rank-sum test. We constructed a multivariable logistic regression model by including each of the conditions as independent variables. The dependent outcome variable was OHSS or not. Receiver operating characteristic (ROC) analysis was carried out and area under the curve (AUC) was compared to evaluate the predicting ability of clinical indicators scores. Based on the cut-off values, sensitivity and specificity were also calculated. A Z test was used for comparing the AUCs between different curves. The Bonferroni method was used adjust for multiple comparisons.

\section{Results}

\section{Comparison baseline characteristics between the OHSS and non-OHSS groups}

A total of 336 cycles were included in this study. According to clinical diagnosis, 61 cycles developed OHSS, with an incidence of $18.15 \%$. Patients were graded according to their clinical symptoms and severity, including 27 cycles of moderate OHSS (8.04\%) and 8 cycles of severe OHSS (2.38\%).

Basal FSH of the OHSS group was lower than the non-OHSS group $(\mathrm{p}<0.001)$. The values of basal LH, basal T, number of AFC, AMH and TSH in the OHSS group were all higher than those in the non-OHSS group, the proportion of PCOS patients in OHSS group was higher than that in control group $(p<0.001)$. There was no significant difference in age, BMI, the duration of infertility, BMI, basal E2 value between these two groups ( $p>0.05$, Table 1$)$.

\section{Clinical parameters between patients with and without OHSS}

The Median of Gn at an initiative dose was about $150 \mathrm{IU}$ in both non-OHSS and OHSS group. The E2 and $P$ on HCG day in the OHSS group was higher than that in the non-OHSS group. The number of oocytes retrieved in the OHSS group was all higher than non-OHSS group $(\mathrm{p}<0.001)$. But the Median of total $\mathrm{Gn}$ dose in the OHSS group was lower than the Non-OHSS, patients with OHSS are more sensitive to Gn. After adjusting age, $\mathrm{AMH}$ and the number of sinus follicles, no statistical difference was found in the 


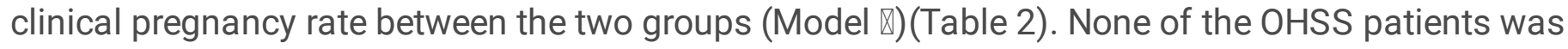
Delayed OHSS.

\section{Logistics regression analysis}

Multiple logistic regression analysis was used to adjust for case-mix differences between OHSS and nonOHSS groups. Basic AMH, E2 on HCG day and the number of eggs obtained were found to be strongly predictive of OHSS (Table 3).

\section{ROC curve}

Cut-off value for predicting the occurrence of OHSS were determined as follows: $A M H \geq 0.75 \mathrm{ng} / \mathrm{mL}, \mathrm{HCG}$ day $E 2 \geq 4824 \mathrm{pg} / \mathrm{mL}$, and egg number $\geq 19.5$, with sensitivities of $77.0 \%, 67.2 \%, 80.3 \%$, and specificity of $68.0 \%, 70.9 \%, 81.5 \%$ respectively. Based on comprehensive judgment, the number of oocytes retrieved has the highest value in predicting the occurrence of OHSS, as shown in Table 4. According to the ROC curve, $\mathrm{AMH}$ value and E2 on HCG day alone predicted the occurrence of OHSS in a medium way, while the number of oocytes retrieved alone predicted the occurrence of OHSS in a high way, with AUC was 0.876 (0.823-0.928, Fig1).

When we combinded the positive indicators including AMH, E2 on HCG day and the number of eggs obtained calculated by regression equation. Regression equation was established as follow:Logit $(\pi)=-8.465+0.085^{\star} \mathrm{AMH}+0.0002^{\star} \mathrm{E} 2$ on $\mathrm{HCG}$ day $+0.266^{\star}$ Number of oocyte retrieved.

The sensitivity of combination indicators was $83.6 \%$, and the specificity was $85.2 \%$, both of which were improved compared with the single index, suggesting that the combination of multiple indicators has a certain significance in predicting the occurrence of OHSS, according to the ROC curve, the area under the curve (AUC) after combining the four indicators is 0.898 (0.854-0.942, Table 4), which strengthens the predictive power and is higher than the AUC for any single indicator to predict the occurrence of OHSS (Fig1).

\section{Discussion}

OHSS is an iatrogenic complication caused by an overreaction to gonadotropin stimulation. OHSS is a common disease during controlled ovarian hyperstimulation treatment, some patients even need to seek initial care in the emergency department because of multiple complications of OHSS, including including ascites, hemodynamic instability, thromboembolic disease, renal insufficiency, acute respiratory distress syndrome, even GDM and neonatal NICU admission and so on[14]. It has been reported in the literature that moderate to severe OHSS accounts for $3-10 \%$ of all ART cycles, and the incidence is as high as $20 \%$ in high-risk women[15]. Although ART is considered safe, but moderate to severe overstimulation syndrome (OHSS) is considerd as a complication have significant morbidity and mortality[16]. Compared with the antagonist protocol, the GnRH-a Prolonged Protocol had higher cumulative live birth rate[8], and 
the incidence of OHSS was not different from that of the antagonist protocol[10]. Due to its high cumulative live birth rate, it is also a commonly used prescription in clinical practice at present. However, there is no study on the risk factors for OHSS in the GnRH-a protocol.

Our study found that the risk of OHSS in patients of GnRH-a Prolonged Protocol was similar to that of other protocol s, including high AMH value, high number of eggs retrieved, and high estrogen! Besides, Young age, lean body mass, Polycystic ovary syndrome (PCOS), Gonadotrophin dosage and so on were consider as risk factors for OHSS[17]. This is the same with our study, younger age also observed in the OHSS group compared to non-OHSS group, but finally, the age factor was not included in the regression analysis, and age could not be used as an indicator to predict the occurrence of OHSS. Because of the harmfulness of OHSS, looking for indicators to predict the occurrence of OHSS can prevent OHSS in advance. As Akanksha et.al reported[18], the optimal thresholds of AMH for predicting OHSS was $22.5 \mathrm{pmol} / \mathrm{L}$, thresholds of AFC was 19.5, and egg numbers was 9.5 , this is similar to our research, which $A M H \geq 4.295 \mathrm{ng} / \mathrm{mL}$, and egg numbers $\geq 18.5$ can predict the occurrence of OHSS. But in their research, peak estradiol levels had no predictive value, this is different from what we found that HCG day E2 $\geq 4824 \mathrm{pg} / \mathrm{mL}$ also can strongly predict the occurrence of OHSS with a sensitivitie of $70.9 \%$. Since the sensitivity of single indicator to predict OHSS was between $70-90 \%$, and the specificity was between $59-88 \%$, we used the combined indicator to predict OHSS, and found that the combined indicator had both good sensitivity (83.6\%) and specificity (85.22\%), which could better predict the occurrence of OHSS.

PCOS also is thought to be an important risk factor of OHSS during ovulation induction[19]. But whether the patient with PCOS can't predict the occurs of severe OHSS in our study, even though the proportion of PCOS patients was higher in the OHSS group, this may be due to the pretreatment we have done in patients with PCOS, such as metformin used in PCOS patient with insulin resistance, has also been studied can reduce the occurrence rate of OHSS[20]. In our study, the number of eggs obtained also can predict OHSS, but the number of eggs obtained is uncontrollable because steering the ovarian response into a supposed optimal range may appear difficult[21], so the number of eggs can't be a reference factor when we formulate the starting dose of Gn for patients, the same with the E2 value on HCG day.

\section{Conclusion}

Patients with serum AMH levels above 7.495ng/ mL, HCG day E2 levels above 4824pg/ mL and egg numbers $\geq 19.5$ can predict OHSS in GnRH-a Prolonged Protocol, but combine these three indicators can predict OHSS better.

\section{Declarations}

\section{Ethics approval and consent to participate}

Not applicable

\section{Consent for publication}


Not applicable

\section{Availability of data and material}

Please contact author for data requests

\section{Funding}

This study was not supported by any funding.

\section{Authors' contributions}

Zexing Yang and Limei He participated in the conception and design of the study. Wen Ya and Meng Rao searched and collected the data, Yuhang Zheng and Limei He performed the analysis, Wen Ya wrote the manuscript. Huawei Wang PhD and Zexing Yang revised the manuscript and gave final approval of this manuscript.

\section{Acknowledgements}

We acknowledge the professional manuscript editing services of Armstrong-Hilton

Ltd.

\section{Conflict of interest}

The authors declare that they have no competing interests.

\section{References}

1. Sansone P, Aurilio C, Pace MC, Esposito R, Passavanti MB, Pota V et al., Intensive care treatment of ovarian hyperstimulation syndrome (OHSS). Ann N Y Acad Sci, 2011,1221: 109-118.

2. Hortu I, Karadadas E, Ozceltik G, Tavmergen E, Tavmergen Goker EN et al., Oxytocin and cabergoline alleviate ovarian hyperstimulation syndrome (OHSS) by suppressing vascular endothelial growth factor (VEGF) in an experimental model. Arch Gynecol Obstet, 2021, 303: 1099-1108.

3. Mourad S, Brown J, Farquhar C., Interventions for the prevention of OHSS in ART cycles: an overview of Cochrane reviews. Cochrane Database Syst Rev, 2017, 1: CD012103.

4. Sun B, Ma Y, Li L, Hu L, Wang F et al., Factors Associated with Ovarian Hyperstimulation Syndrome (OHSS) Severity in Women With Polycystic Ovary Syndrome Undergoing IVF/ICSI. Front Endocrinol (Lausanne), 2020, 11: 615957.

5. Kuroda K, Nagai S, Ikemoto Y, Matsumura Y, Ochiai A et al., Incidences and risk factors of moderateto-severe ovarian hyperstimulation syndrome and severe hemoperitoneum in 1,435,108 oocyte retrievals. Reprod Biomed Online, 2021,42:125-132. 
6. Wu HD, Song ZK, Xu XY, Cao HY, Wei Q et al., Combination of D-dimer and simplified pulmonary embolism severity index to improve prediction of hospital death in patients with acute pulmonary embolism. J Int Med Res, 2020,48:300060520962291.

7. Tölle A, Jung K, Friedersdorff F, Maxeiner A, Lein M et al., The discriminative ability of Prostate Health Index to detect prostate cancer is enhanced in combination with miR-222-3p. Cancer Biomark, 2021,30: 381-393.

8. Liu L, Xu Y, Huang J, Zhou C. Patients with higher anti-Mullerian hormone levels from POSEIDON group 4 benefit from $\mathrm{GnRH}$-agonist long protocol: A retrospective study. Eur J Obstet Gynecol Reprod Biol, 2021, 257: p. 88-94.

9. Yang J, Zhang $X$, Ding $X$, Wang $Y$, Huang $G$ et al., Cumulative live birth rates between $\mathrm{GnRH}$-agonist long and $\mathrm{GnRH}$-antagonist protocol in one ART cycle when all embryos transferred: real-word data of 18,853 women from China. Reprod Biol Endocrinol, 2021, 19: 124.

10. Alhilali MJ, Parham A, Attaranzadeh A, Amirian M, Azizzadeh M. Prognostic role of follicular fluid tumor necrosis factor alpha in the risk of early ovarian hyperstimulation syndrome. BMC Pregnancy Childbirth, 2020,20: 691.

11. Rotterdam, E.A.-S.P.c.w.g., Revised 2003 consensus on diagnostic criteria and long-term health risks related to polycystic ovary syndrome (PCOS). Hum Reprod, 2004,19:41-47.

12. Practice Committee of the American Society for Reproductive Medicine. Electronic address, A.a.o. and M. Practice Committee of the American Society for Reproductive, Prevention and treatment of moderate and severe ovarian hyperstimulation syndrome: a guideline. Fertil Steril, 2016,106:16341647.

13. Yang R, Guan Y, Perrot V, Ma J, Li R et al., Comparison of the Long-Acting GnRH Agonist Follicular Protocol with the GnRH Antagonist Protocol in Women Undergoing In Vitro Fertilization: A Systematic Review and Meta-analysis. Adv Ther, 2021,38:2027-2037.

14. Hu L, Xie R, Wang M, Sun Y. Patients with IVF complicated by moderate-to-critical OHSS experience increased thrombosis, GDM and neonatal NICU admission but slightly shorter gestation compared with matched IVF counterparts: A retrospective Chinese cohort study. Reprod Biol Endocrinol, 2021,19:8.

15. Nastri CO, Teixeira DM, Moroni RM, Leitão VM, Martins WP. Ovarian hyperstimulation syndrome: pathophysiology, staging, prediction and prevention. Ultrasound Obstet Gynecol, 2015,45: 377-393.

16. Liu F, Jiang Q, Sun X, Huang Y, Zhang Z et al. Lipid Metabolic Disorders and Ovarian Hyperstimulation Syndrome: A Retrospective Analysis. Front Physiol, 2020,11:491892.

17. Delvigne A, Rozenberg S. Epidemiology and prevention of ovarian hyperstimulation syndrome (OHSS): a review. Hum Reprod Update, 2002,8:559-577.

18. Sood A, Goel A, Boda S, Mathur R. Prediction of significant OHSS by ovarian reserve and ovarian response - implications for elective freeze-all strategy. Hum Fertil (Camb), 2020,24:1-7.

19. Namavar Jahromi B MD, Parsanezhad ME MD, Shomali Z MD, Bakhshai P MD, Alborzi M MD, Moin Vaziri N MD PhD, Anvar Z PhD. Ovarian Hyperstimulation Syndrome: A Narrative Review of Its 
Pathophysiology, Risk Factors, Prevention, Classification, and Management. Iran J Med Sci, 2018,43:248-260. PMID: 29892142, PMCID: PMC5993897.

20. Mascarenhas $\mathrm{M}$, Balen $\mathrm{AH}$. The high responder: a review of pathophysiology and outcomes during IVF treatment. Hum Fertil (Camb), 2017,20:155-167.

21. Broekmans FJ. Individualization of FSH Doses in Assisted Reproduction: Facts and Fiction. Front Endocrinol (Lausanne), 2019,10: 181.

\section{Tables}

Table1.Baseline characteristics of patients.

\begin{tabular}{|c|c|c|c|}
\hline & $\begin{array}{l}\text { Non-OHSS } \\
(n=275)\end{array}$ & $\begin{array}{l}\text { OHSS } \\
(n=61)\end{array}$ & $P$ value \\
\hline Age (years) & $29.75 \pm 4.30$ & $28.77 \pm 3.81$ & 0.101 \\
\hline $\mathrm{BM} \triangle \mathrm{kg} / \mathrm{m}^{2} \square$ & $23.13 \pm 3.49$ & $23.23 \pm 3.71$ & 0.838 \\
\hline Duration of infertility (years) & $3.00(2.00,5.00)$ & $3.00(2.00,4.00)$ & 0.232 \\
\hline Basal FSH ${ }^{*}(\mathrm{mlU} / \mathrm{mL})$ median (IQR) & $6.29(5.46,7.27)$ & $5.62(4.93,6.48)$ & 0.001 \\
\hline Basal LH ${ }^{\star}(\mathrm{mlU} / \mathrm{mL})$ median (IQR) & $6.87(5.02,10.72)$ & $8.60(6.90,12.77)$ & 0.003 \\
\hline Basal $\mathrm{E}_{2}(\mathrm{pg} / \mathrm{mL})$ median (IQR) & $37.71(32.72,46.73)$ & $37.46(31.95,47.73)$ & 0.876 \\
\hline Basal $T^{*}(\mathrm{ng} / \mathrm{mL})$ median (IQR) & $0.32(0.23,0.44)$ & $0.39(0.28,0.54)$ & 0.001 \\
\hline $\mathrm{AFC}^{*}$ median (IQR) & $22.00(15.00,24.00)$ & $24.00(24.00,24.00)$ & $<0.001$ \\
\hline $\mathrm{AMH}^{*}(\mathrm{ng} / \mathrm{mL})$ & $5.60(3.22,8.89)$ & $11.19(7.55,15.96)$ & $<0.001$ \\
\hline $\mathrm{TSH}^{*}(\mathrm{mU} / \mathrm{L})$ & $2.33(1.65,3.42)$ & $3.10(2.07,3.93)$ & 0.015 \\
\hline PCOS (\%) & $125 \rrbracket 45.5 \% \rrbracket$ & $51 \rrbracket 83.6 \% \rrbracket$ & $<0.001$ \\
\hline
\end{tabular}

$>$

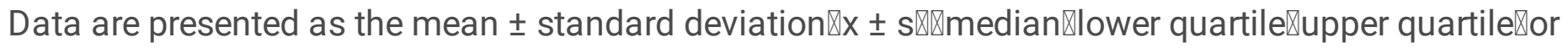
count (percentage) , BMI body mass index, AFC antral follicle count, TSH thyroid stimulating hormone, FSH follicle-stimulating hormone, LH luteinizing hormone, E2 estrogen, T androgen, IQR interquartile range.

$\mathrm{t}$ value had been corrected, ${ }^{\star} \mathrm{p}<0.05$ 
Table 2. Clinical parameters and outcomes between non-OHSS and OHSS group.

\begin{tabular}{|c|c|c|c|}
\hline & $\begin{array}{l}\text { Non-OHSS } \\
(n=275)\end{array}$ & $\begin{array}{l}\text { OHSS } \\
(n=61)\end{array}$ & $\mathrm{p}$ value \\
\hline $\begin{array}{l}\text { durations of } \mathrm{Gn}(\mathrm{IU}) \text { median } \\
(\mathrm{IQR})^{\star}\end{array}$ & $12.00 \otimes 11.00,13.00 \rrbracket$ & $11.00 \otimes 10.50,12.00 \otimes$ & 0.003 \\
\hline initiative dose of $\mathrm{Gn}(\mathrm{IU})$ * & $150.00 \rrbracket 150.00,225.00 \rrbracket$ & 150.00ه150.00,200.00》 & 0.034 \\
\hline total Gn dose (IU)* & $\begin{array}{l}2375.00 \\
₫ 1775.00,3150.00 \rrbracket\end{array}$ & 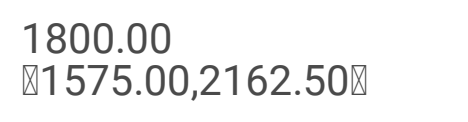 & $<0.001$ \\
\hline HCG dose (IU) & $\begin{array}{l}5000.00 \\
₫ 4000.00,5000.00 \rrbracket\end{array}$ & $\begin{array}{l}5000.00 \\
\square 3500.00,5000.00 \rrbracket\end{array}$ & 0.636 \\
\hline E2 on $\mathrm{HCG}(\mathrm{pg} / \mathrm{mL})$ * & 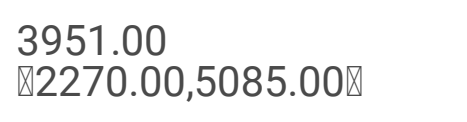 & 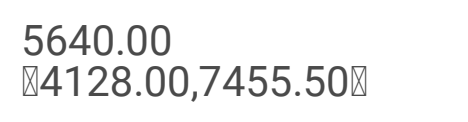 & $<0.001$ \\
\hline P on HCG $(\mathrm{ng} / \mathrm{mL})^{*}$ & $0.78 ه 0.50,1.20 \rrbracket$ & $1.07 \rrbracket 0.70,1.27 \rrbracket$ & 0.014 \\
\hline LH on HCG $(\mathrm{mlU} / \mathrm{mL})$ & $0.95 \rrbracket 0.59,1.36 \rrbracket$ & $0.83 \varangle 0.58,1.13 \rrbracket$ & 0.141 \\
\hline Number of oocytes retrieved * & $15.00 \otimes 11.00,19.00 \rrbracket$ & $25.00 \rrbracket 20.00,28.00 \rrbracket$ & $<0.001$ \\
\hline $\begin{array}{l}\text { clinical cumulative pregnancy } \\
\text { rates }\end{array}$ & OR Value & & $\begin{array}{l}\mathrm{P} \\
\text { Value }\end{array}$ \\
\hline Model $\mathbb{Z}$ & 3.068 & & 0.014 \\
\hline 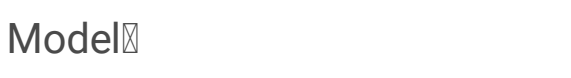 & 2.837 & & 0.023 \\
\hline Model $\otimes$ & 2.308 & & 0.073 \\
\hline
\end{tabular}

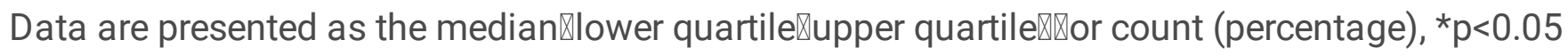

Model『: The occurrence of OHSS was the independent variable $(0=$ non-OHSS, $1=\mathrm{OHSS})$, and clinical pregnancy was used as the dependent variable $(0=$ not pregnancy, $1=$ pregnancy $)$, Model $\nabla$ on the basis of the model囚adjusted for age, Model $₫$ On the basis of the model $\otimes$ adjusted for Age, AMH, AFC.

Table 3. Coefficient estimates of the logistic regression to predict OHSS

\begin{tabular}{llll} 
explanatory variables & B & Standard error & P value \\
\hline AMH $(\mathrm{ng} / \mathrm{mL})$ & 0.085 & 0.038 & 0.027 \\
\hline E2 on HCG day $(\mathrm{pg} / \mathrm{mL})$ & $<0.001$ & $<0.001$ & 0.013 \\
\hline Number of retrieved oocytes & 0.266 & 0.040 & $<0.01$ \\
\hline Constant term & -8.465 & 0.977 &
\end{tabular}


Table 4. Statistical data of receiver-operating characteristics curve comparisons of different parameters in predicting OHSS

\begin{tabular}{|c|c|c|c|c|}
\hline & AUC (95\% Cl) & Cut-off value & Specið\%区 & Sensið\%区 \\
\hline $\mathrm{AMH}(\mathrm{ng} / \mathrm{mL})$ & $0.754 \rrbracket 0.691-0.817 \rrbracket$ & 7.495 & 77.0 & 68.0 \\
\hline E2 on HCG day (pg/ mL) & $0.738 \otimes 0.667-0.809 \rrbracket$ & 4824 & 67.2 & 70.9 \\
\hline Number of oocytes retrieved & $0.876 \rrbracket 0.823-0.928 \rrbracket$ & 19.5 & 80.3 & 81.5 \\
\hline Combination index & $0.898 \otimes 0.854-0.942 \rrbracket$ & & 85.2 & 83.6 \\
\hline
\end{tabular}

\section{Figures}

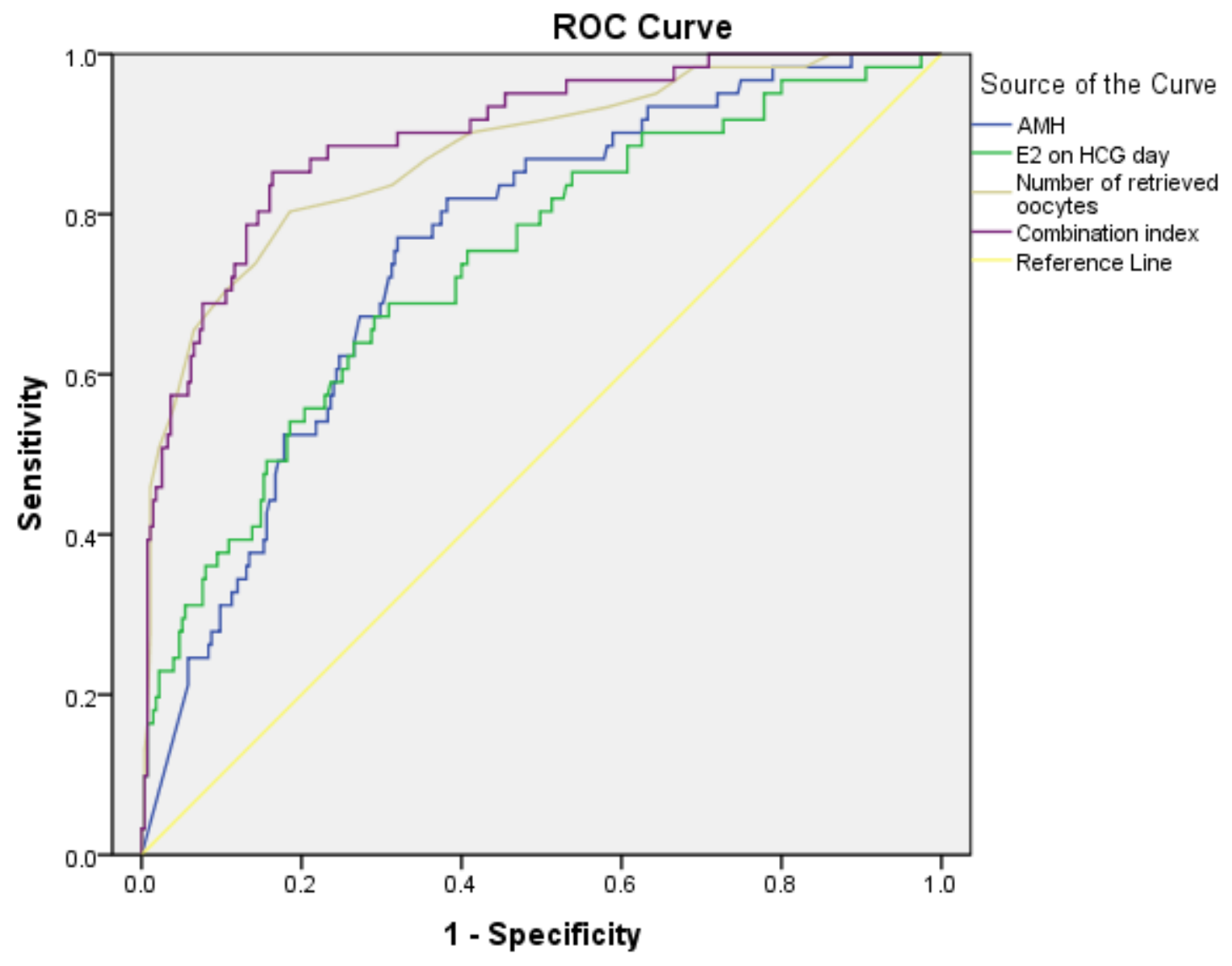

Diagonal segments are produced by ties.

\section{Figure 1}

Receiver-operating characteristics curve comparisons of different parameters in predicting OHSS. 